\title{
Craniofacial Bone Regeneration using iPS Cell-Derived Neural Crest Like Cells
}

\author{
Kazuko Kikuchi $^{1,2)}$, Tomoyuki Masuda ${ }^{2)}$, Naoki Fujiwara ${ }^{2)}$, Akiyoshi Kuji ${ }^{1)}$, Hiroyuki Miura ${ }^{3)}$, Han-Sung Jung ${ }^{4,5)}$, \\ Hidemitsu Harada ${ }^{2)}$ and Keishi Otsu' ${ }^{2)}$
}

\author{
I) Division of Pediatric and Special Care Dentistry, Department of Oral Health Science, School of Dentistry, Iwate Medical University, Morioka, Japan \\ 2) Division of Developmental Biology and Regenerative Medicine, Department of Anatomy, Iwate Medical University, Yahaba, Japan \\ 3) Division of Dental Education, Department of Oral Medicine, School of Dentistry, Iwate Medical University, Morioka, Japan \\ 4) Division in Anatomy and Developmental Biology, Department of Oral Biology, Oral Science Research Center, BK21 PLUS Project, Yonsei University \\ College of Dentistry, Seoul, South Korea \\ 5) Oral Biosciences, Faculty of Dentistry, The University of Hong Kong, Hong Kong SAR, People's Republic of China \\ (Accepted for publication, September 5, 2017)
}

\begin{abstract}
Induced pluripotent stem (iPS) cells represent a powerful source for cell-based tissue regeneration because they are patient-specific cells and can differentiate into specialized cell types. Previously, we have demonstrated the derivation of neural crest like cells from iPS cells (iPS-NCLCs), and these cells have the potential to differentiate into dental mesenchymal cells, which subsequently differentiate into odontoblasts and dental pulp cells. In this study, we show that iPS-NCLCs can differentiate into mesenchymal stem cells (iPS-NCLC-MSCs), which contribute to craniofacial bone regeneration. iPS-NCLCs were cultured in serum-containing media and differentiated into functional MSCs, as confirmed by expression MSC markers and their ability to differentiate into osteoblasts, adipocytes, and chondrocytes in vitro. iPSNCLC-MSCs were negative for markers of undifferentiated iPS cells and did not develop into teratomas when transplanted to immunodeficient mice. Further, iPS-NCLC-MSCs grew normally and differentiated into osteoblasts on hydroxyapatite scaffolds in vitro. To assess the potential of iPS-NCLC-MSCs to regenerate craniofacial bone in vivo, iPS-NCLC-MSCs were transplanted into critical-size calvarial defects in immunodeficient mice for 8 weeks. Histological analysis revealed that iPS-NCLC-MSCs differentiated into osteoblasts and contributed to bone regeneration without tumor formation. These results indicate that iPS-NCLC-MSCs could be a potential candidate for cell-based craniofacial bone tissue repair and regeneration.
\end{abstract}

Key words: Craniofacial bone regeneration, Induced pluripotent stem cells (iPSCs), Neural crest cells, Mesenchymal stem cells, Osteoblasts

\section{Introduction}

Numerous approaches have been proposed for bone defect repair and regeneration. To date, autogenous bone grafting of defects have been regarded as the "gold standard" because it has osteoconductive and osteoinductive properties and is a source of osteoprogenitor cells $\mathrm{s}^{1)}$. However, harvesting bone grafts often has disadvantages, including limited availability and donor site morbidity, additional surgery under general anesthesia, and risk of postoperative pain ${ }^{2,3)}$. Further, because allogeneic and xenogeneic grafts also have limited utility owing to the risks of immune rejection and infectious disease ${ }^{4)}$, alternative treatment approaches would be advantageous. To overcome these limitations, bone tissue engineering using stem cells has recently attracted wide attention and shows promise in treating bone defects and avoiding bone graft problems.

In recent years, multipotent mesenchymal stem cells (MSCs), which can be isolated from adult tissues, have emerged as a promising cell source for tissue regeneration including bone. MSCs have been shown to possess a self-renewal capacity and the potential to differentiate into various mesenchymal cell lineages including osteoblasts, chondrocytes, and adipocytes, both in vitro and in vivo, as well as their potent paracrine anti-inflammatory properties ${ }^{5-7)}$. Conversely, there are factors that limit the routine use of adult tissue-derived MSCs for clinical Correspondence to: Dr. Keishi Otsu, Division of Developmental Biology and Regenerative Medicine, Department of Anatomy, Iwate Medical University, 2-1-1 Nishitokuta, Yahaba, Iwate, 028-3694 Japan; Phone: +81 19651 5111; Fax: +81 19 908 -8017; E-mail address: kotsu@iwate-med.ac.jp treatment. For instance, the procedures involved in harvesting MSCs are invasive, expensive, and laborious. MSCs also have a limited proliferative and differentiation capacity that significantly decreases with age $\mathrm{e}^{8)}$, making it difficult to obtain the cell numbers required for transplantation $^{9-11)}$. Therefore, an alternative, unlimited source of MSCs derived from pluripotent stem cells in order to obtain larger populations would address these limitations.

Induced pluripotent stem (iPS) cells are generated by introducing defined transcription factors such as Sox2, Klf4, Oct3/4, and cMyc (called Yamanaka factors) into somatic cells, which have multipotential ability to differentiate into cell types characteristic of the 3 germ layers of embryonic stem cells (ESCs) in vitro and in vivo. This technology allows us to obtain pluripotent stem cells from patients' own cells and avoid the immune rejection and ethical issue of ESCs. Additionally, iPS cells offer an advantage over adult stem cells as they exhibit unlimited growth capacity as an inexhaustible stem cell source. Thus, iPS cells provide a powerful tool for stem cell-based regenerative medicine.

Recently, we have established a culture protocol to induce neural crest-like cells from iPS cells (iPS-NCLCs) and demonstrated that they have the potential to differentiate into dental mesenchymal cell-derived odontoblasts and dental pulp cells to form tooth structures ${ }^{12,13)}$. Based on results showing pluripotency of iPS-NCLCs, we hypothesized that iPS-NCLCs could differentiate into MSCs and become a suitable cell source for the regeneration of craniofacial bone that originates from neural crest-derived $\mathrm{MSCs}^{14-16)}$. Toward this theory, we generated 
MSCs from iPS-NCLCs (iPS-NCLC-MSCs) and evaluated their molecular characteristics and tumorigenicity. Then, the differentiation potential of iPS-NCLC-MSCs into osteoblasts was investigated in vitro. Finally, we created a calvarial defect model and assessed the efficacy of iPS-NCLC-MSCs for bone regeneration in vivo. In the present study, we propose a novel stem cell source for personalized and functional craniofacial bone repair.

\section{Antibodies}

\section{Materials and Methods}

Anti-STRO-1, osteopontin (OP), fatty acid-binding protein (FABP), and collagen type II (Col2) antibodies were purchased from R\&D systems (Minneapolis, MN). Anti-Gli-1, c-Myc, Osterix (OSX), and bone sialoprotein (BSP) antibodies were obtained from Abcam (Cambridge, U.K.). Anti-Osteonectin (ON) antibody was from Cosmo Bio (Tokyo, Japan). Anti-green fluorescent protein (GFP) monoclonal antibodies were a product of Cell Signaling Technology (Danvers, MA). DAPI, Alexa Fluor 488, and Alexa Fluor 546 secondary antibodies were from Thermo Fisher Scientific (Tokyo, Japan).

\section{Animals}

The 8-week-old male KSN/Slc nude mice were obtained from Japan SLC, Inc. (Shizuoka, Japan) and maintained in plastic cages in ambient temperature of $23 \pm 1^{\circ} \mathrm{C}$ with ad libitum access to water and a standard laboratory pellet diet. The experiments were conducted in accordance with the Protocols for the Humane Treatment of Animals of Iwate Medical University. The protocol of the animal experiment was approved by the Institutional Animal Care and Use Committee of Iwate Medical University, \#27-044 and the Recombinant DNA Experiment Committee of Iwate Medical University, \#389.

\section{Cell culture and MSC induction}

Mouse iPS cell line (iPS-MEF-Ng-20D-17, iPS-Stm-FB/gfp-991, iPS-Hep-FB/Ng/gfp-103C-1: RIKEN BRC, Japan) ${ }^{17)}$ were cultured on mitotically inactivated mouse embryonic fibroblasts in the culture medium: Dulbecco's modified Eagle's medium (DMEM, Thermo Fisher Scientific, Waltham, MA) containing 15\% fetal bovine serum (FBS), $0.1 \mathrm{mM}$ non-essential amino acid (Thermo Fisher Scientific), $0.1 \mathrm{mM}$ 2-Mercaptoethanol (Sigma-Aldrich, Tokyo, Japan), $50 \mathrm{U} / \mathrm{ml}$ and $50 \mu \mathrm{g} /$ $\mathrm{ml}$ Penicillin-Streptomycin (Thermo Fisher Scientific) supplemented with $1000 \mathrm{U} / \mathrm{ml}$ mouse leukemia inhibitory factor (LIF, Chemicon, Billerica, MA). The cultures were maintained at $37{ }^{\circ} \mathrm{C}$ in a $5 \% \mathrm{CO}_{2}$ humidified incubator and the medium was changed every other day.

For iPS-NCLC-MSCs derived from iPS cells, we modified the previously described methods ${ }^{12}$. Briefly, iPS cells were dissociated by $0.25 \%$ Trypsin/EDTA and transferred to neural induction medium for suspension culture in a non-adherent culture dish (HydroCell, CellSeed, Tokyo, Japan) to form neuroectodermal spheres. The neural induction medium consisted of a 1:1 ratio of DMEM/F12 (Thermo Fisher Scientific) and Neurobasal Medium (Thermo Fisher Scientific) supplemented with $0.5 \times \mathrm{N}-2$ (Thermo Fisher Scientific), $0.5 \times$ B27 (Thermo Fisher Scientific), $5 \mu \mathrm{g} / \mathrm{ml}$ insulin (Sigma-Aldrich), $20 \mathrm{ng} /$ $\mathrm{ml}$ bFGF (R\&D Systems), $20 \mathrm{ng} / \mathrm{ml}$ EGF (R\&D Systems), and $50 \mathrm{U} /$ $\mathrm{ml}$ and $50 \mu \mathrm{g} / \mathrm{ml}$ Penicillin-Streptomycin. After 4 days of suspension culture, the spheres were transferred to a fibronectin-coated culture dish (BD Biosciences, San Jose, CA). After 8-10 days, the spheres or rosettes were manually dissected away and the resultant iPSNCLCs were passaged with $0.25 \%$ Trypsin-EDTA and replated on a fibronectin culture dish. After checking the expression of NC marker as previously described ${ }^{12)}$, iPS-NCLCs were cultured and maintained in DMEM containing 10\% FBS and $50 \mathrm{U} / \mathrm{ml}$ and $50 \mu \mathrm{g} / \mathrm{ml}$ PenicillinStreptomycin on CorningTM PrimariaTM tissue culture dishes (Thermo Fisher Scientific) to induce MSCs.

For iPS-NCLC-MSCs cultured on hydroxyapatite (HA), unidirectional porous HA scaffolds ${ }^{18-20)}$ (Kurarife, Kuraray Medical Inc., Tokyo, Japan) were coated with fibronectin overnight at $4^{\circ} \mathrm{C}$ and washed with PBS 3 times. Dissociated iPS-NCLC-MSCs were seeded on the HA scaffolds and cultured in DMEM supplemented with $10 \%$ FBS for 2 days before osteoinduction.

\section{Flow cytometric analysis}

The cells were detached with $0.25 \%$ Trypsin-EDTA. For cellular staining, approximately $0.5-1 \times 10^{6}$ cells were incubated with PEconjugated primary antibody (anti-CD44, CD29, Sca-1, CD90.2, CD45, and CD11b; Miltenyi Biotec Inc., Auburn, CA,; BioLegend, San Diego, $\mathrm{CA}$ ) for $30 \mathrm{~min}$ on ice. Cells were analyzed using a flow cytometer EPICS XL ADC (Beckman Coulter, Tokyo, Japan) and PERFLOW Sort V-4cTS2L (Furukawa Electric, Tokyo, Japan) with the accompanying software.

\section{Immunostaining}

Indirect immunostaining was performed on cultured cells and paraffin-embedded and frozen sections as previously described ${ }^{12,21)}$. After blocking with $5 \%$, the cells and tissue sections were reacted with the above antibodies overnight at $4{ }^{\circ} \mathrm{C}$. After administration of secondary antibodies for $30 \mathrm{~min}$, fluorescence images were obtained using a fluorescence microscope (IX71, BX51 Olympus) or laserscanning confocal microscope (FV300, Olympus). Control specimens were incubated directly with secondary antibodies in the absence of primary antibodies and processed as above. No significant positive immunoreactivity was found in controls. Image analyses were carried out using ImageJ or the software provided with the confocal microscope. All imaging data were obtained from experiments that were replicated at least 3 times.

\section{Differentiation into mesenchymal lineage cells}

Differentiation capacity of the iPS-NCLC-MSCs was investigated using the Mouse Mesenchymal Stem Cell Functional Identification Kit (R\&D systems) according to the manufacturer's protocol. Briefly, for osteogenic differentiation, cells were incubated in osteogenic differentiation medium for 3 weeks and detected by alizarin red staining and immunostaining of OP. For adipogenic differentiation, cells were incubated in adipogenic differentiation medium for 2 weeks and detected by oil red staining and immunostaining for FABP. For chondrogenic differentiation, cells were cultured in the conical tube with chondrogenic differentiation medium for 3 weeks to form pellets, which were fixed and prepared for frozen section for alcian blue staining and Col2 immunostaining.

\section{Teratoma formation}

We followed our previously described methods ${ }^{12)}$ in which undifferentiated iPS cells and iPS-NCLC-MSCs $\left(1 \times 10^{6}\right.$ cells $)$ were mixed in type I-A collagen $(500 \mu$ l) (Cellmatrix, Nitta Gelatin, Osaka, Japan) and were subcutaneously injected into the lower flank of agematched nude mice. The 4 mice injected with undifferentiated iPS cells and 4 mice injected with iPS-NCLC-MSCs were sacrificed after 4 weeks of cell injection.

\section{Real-time RT-PCR}

We followed our previously described methods ${ }^{12,21)}$ in which 
Kazuko Kikuchi et al.: Bone Regeneration by iPS-Derived Neural Crest Cells

Table 1 qRT-PCR Oligonucleotide Primers Used to Determine the Relative RNA Expression Level.

\begin{tabular}{|c|c|c|}
\hline mRNA & Orientation & Sequence \\
\hline \multirow[t]{2}{*}{ Nanog } & Forward & 5'-GCCTCCAGCAGATGCAAGAAC-3' \\
\hline & Reverse & 5'-CTGCAATGGATGCTGGGATAC-3' \\
\hline \multirow[t]{2}{*}{ Lin28a } & Forward & 5'-GTCCAGGCATGGGAACATGA-3' \\
\hline & Reverse & 5'-GGCAGGACACTTGCCACTGA-3' \\
\hline \multirow[t]{2}{*}{ Oct3/4 } & Forward & 5'-CAGACCACCATCTGTCGCTTC-3' \\
\hline & Reverse & 5'-AGACTCCACCTCACACGGTTCTC-3' \\
\hline \multirow[t]{2}{*}{ Klf4 } & Forward & 5'-AAGGCACACCTGCGAACTCA-3' \\
\hline & Reverse & 5'-TTTGCGGTAGTGCCTGGTCA-3' \\
\hline \multirow[t]{2}{*}{ Sox 2} & Forward & 5'-AACCGATGCACCGCTACGA-3' \\
\hline & Reverse & 5'-TGCTGCGAGTAGGACATGCTG-3' \\
\hline \multirow[t]{2}{*}{ OSX } & Forward & 5'-AAGTTATGATGACGGGTCAGGTACA-3' \\
\hline & Reverse & 5'- AGAAATCTACGAGCAAGGTCTCCAC-3' \\
\hline \multirow[t]{2}{*}{ BMP2 } & Forward & 5'-TGACTGGATCGTGGCACCTC-3' \\
\hline & Reverse & 5'-CAGAGTCTGCACTATGGCATGGTTA-3' \\
\hline \multirow[t]{2}{*}{ Runx2 } & Forward & 5'-GCACAAACATGGCCAGATTCA-3' \\
\hline & Reverse & 5'-AAGCCATGGTGCCCGTTAG-3' \\
\hline \multirow[t]{2}{*}{ Col1a2 } & Forward & 5'-CTTCTGCAGGGTTCCAACGA-3' \\
\hline & Reverse & 5'-AATGTCCAGAGGTGCAATGTCAA-3' \\
\hline \multirow[t]{2}{*}{ ALP } & Forward & 5'-GCAGTATGAATTGAATCGGAACAAC-3' \\
\hline & Reverse & 5'-ATGGCCTGGTCCATCTCCAC-3' \\
\hline \multirow[t]{2}{*}{ PPAR $\gamma$} & Forward & 5'-GGAGCCTAAGTTTGAGTTTGCTGTG-3' \\
\hline & Reverse & 5'-TGCAGCAGGTTGTCTTGGATG-3’' \\
\hline \multirow[t]{2}{*}{ Col2a1 } & Forward & 5'-CATCCAGGGCTCCAATGATGTA-3' \\
\hline & Reverse & 5'-ATGTCCATGGGTGCGATGTC-3' \\
\hline \multirow[t]{2}{*}{ GAPDH } & Forward & 5’-TGTGTCCGTCGTGGATCTGA-3' \\
\hline & Reverse & 5'-TTGCTGTTGAAGTCGCAGGAG-3' \\
\hline
\end{tabular}

total RNA was isolated using an RNeasy mini Kit (Qiagen, Tokyo, Japan) and reverse transcription of total RNA was performed using a PrimeScript RT reagent kit (Takara Bio, Siga, Japan). cDNA amplification was performed in quantitative reverse transcription polymerase chain reaction (qRT-PCRs) using SYBR ${ }^{\circledR}$ Premix Ex Taq $^{\mathrm{TM}}$ (Takara Bio) with a Thermal Cycler Dice (Takara Bio) according to the manufacturer's protocol. Primer sequences for each cDNA are listed in Table 1. Target gene expression levels were normalized based on GAPDH expression. The relative gene expression levels were calculated based on control culture levels using the comparative $\mathrm{Ct}$ (2$\Delta \Delta \mathrm{Ct}$ ) method. Experiments were carried out in triplicate.

\section{Calvarial defects and surgical procedure}

We followed our previously described method ${ }^{22)}$ of using a trephine bar (Sophia Tech, Inc., Tokyo, Japan) and saline irrigation to create unilateral full-thickness calvarial defects $(2.4 \mathrm{~mm}$ diameter) in the nonsuture-associated right and left parietal bone under general anesthesia. The dispersed GFP-expressing iPS-NCLC-MSCs $\left(1 \times 10^{6}\right.$ cells $)$ were transplanted with $\beta$-tricalcium phosphate $\left(\beta\right.$-TCP; OSferion ${ }^{\circledR}$, Olympus, Tokyo, Japan) into the defects and the skin and periosteum were restored and sewed. As $\geq 2 \mathrm{~mm}$ was accepted as the critical size for the mouse calvarial defect ${ }^{23)}$, we confirmed that a defect $2.4 \mathrm{~mm}$ in size irrigated using normal saline did not demonstrate significant radiographic or histological evidence of spontaneous healing after 12 weeks ${ }^{22}$.

\section{Analysis of bone regeneration}

Tissues from the original defect area of each group were fixed in $4 \%$ PFA at $4{ }^{\circ} \mathrm{C}$ overnight and decalcified in $10 \%$ EDTA, and paraffin- embedded frontal cross-sections were made. Hematoxylin and eosin (H\&E) staining was performed on every 10th section throughout the sample to examine the histological features in a detailed manner, and newly formed bone was evaluated for each group. Immunostaining for bone marker was performed as described above.

\section{Statistics}

All data are reported as mean \pm SD. Statistical significance was assessed using two-tailed Student $t$ tests for 2 groups or analysis of variance Tukey test for $>2$ groups. Significance was defined as $\mathrm{P}<0.05$.

\section{Results}

\section{Induction of differentiation from iPS cells to mesenchymal stem cells} through neural crest like cells

Previously, we have established an efficient culture method for induction of neural crest-like cells derived from mouse iPS cells (iPSNCLCs) ${ }^{12}$. First, mouse iPS cells (Fig. 1A) were cultured in suspension for 4 days in neural crest induction medium to produce a neural sphere (Fig. 1B). Next, the neural spheres spontaneously adhered to the bottom of the dish in 1-2 days and the rosette-like structures gave rise to stellate-morphology migratory cells (Fig. 1C and D). The neural spheres were removed from the rosette, and the migratory cells were passaged and cultured (Fig. 1E). The cells were positive for neural crest markers such as AP2- $\alpha, \mathrm{p} 75^{\mathrm{NTR}}$, and Wnt- $1^{12)}$, indicating neural crest cells. Subsequently, when the cells were cultured in serum-containing medium, they morphologically changed and exhibited a spindle shape like MSCs (Fig. 1F). Immunocytochemistry showed that they were positive for MSC markers, Gli-1 (Fig. 1G) and STRO-1 (Fig. 1H). Flow cytometric analysis revealed that the cells were also positive for CD44 and CD29 and partially expressed Sca-1 and CD90.2 (Fig. 2). In contrast, they were negative for CD45 and CD11b, leukocyte and macrophage markers, respectively (Fig. 2), indicating that iPS-NCLCs are established as having a potential for differentiation into MSCs.

\section{iPS-NCLC-MSCs lost tumorigenicity}

We have previously demonstrated that iPS-NCLCs lost tumorigenicity in vivo, which undifferentiated iPS cells possess ${ }^{12}$. Thus, in order to confirm that iPS-NCLC-MSCs also lost tumorigenicity, we implanted iPS-NCLC-MSCs subcutaneously into nude mice. Four weeks post-implantation, while undifferentiated iPS cells formed teratoma subcutaneously (Fig. 3 left), iPS-NCLC-MSCs did not form any visible tumors (Fig. 3 right). Quantitative PCR to examine the gene expression associated with maintaining an undifferentiated state and tumorigenicity revealed that Nanog, Lin28a, Oct3/4, and klf-4 were remarkably decreased in iPS-NCLC-MSCs compared to undifferentiated iPS cells, while Sox2 was unchanged (Fig. 4). Immunofluorescence showed that c-Myc was not expressed in iPS-NCLC-MSCs (data not shown). These results demonstrated that iPS-NCLC-MSCs induced from our culture protocol had no tumorigenicity.

\section{iPS-NCLC-MSCS have the capacity to differentiate into multiple mesenchymal lineage cells}

To verify the multipotency of iPS-NCLC-MSCs, in vitro differentiation into osteogenic, adipogenic, and chondrogenic lineages was performed using cell-lineage specific induction culture system. After 3 weeks of osteoinduction culture, mRNA expression of genes related to osteogenesis, such as OSX, bone morphogenetic protein 2 (BMP2), runt-related transcription factor 2 (Runx2), collagen type I, alpha 2 (Col1a2), and alkaline phosphatase (ALP) remarkably increased in osteoinduced iPS-NCLC-MSCs compared to undifferentiated iPS 
J.Hard Tissue Biology Vol. 27(1): 1-10, 2018
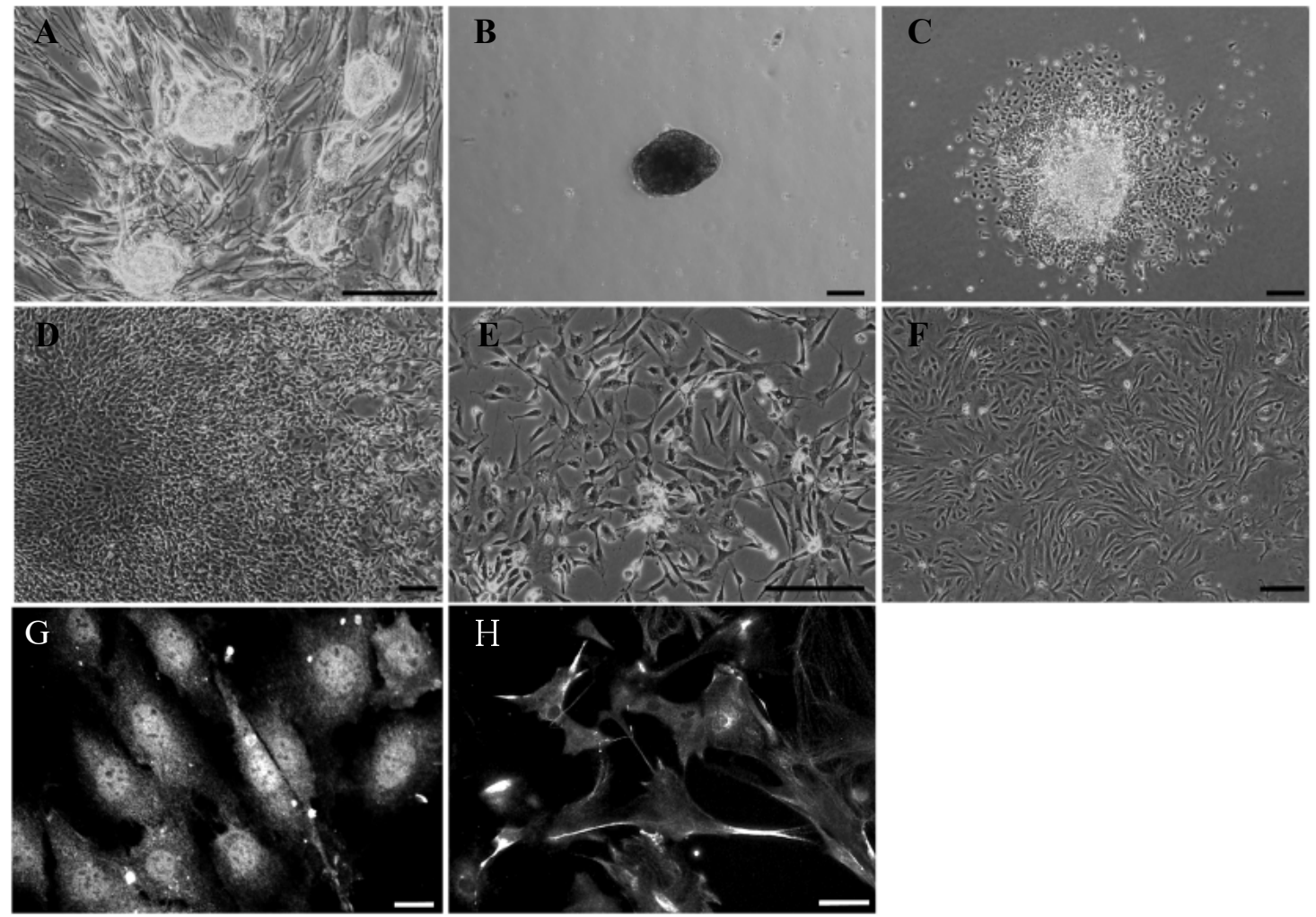

Figure 1. Derivation of MSCs from iPS cells via neural crest cells. Morphological overview of MSCs derivation (A-F). miPS cells (A) were differentiated in suspension culture to form spheres (B), which spontaneously attached and formed rosette-like structures and stellate morphology cells migrating away in neural crest differentiation medium (C). Rosettes were removed (D) and the migrating cells formed a uniform cell population (E). The cells were then cultured in serum-containing medium and exhibited spindle-shaped morphology (F). Immunofluorescence of Gli-1 (G) and STRO-1 (H) in iPS-NCLC-MSCs. Scale bar = $200 \mu \mathrm{m}(\mathrm{A}-\mathrm{F}), 20 \mu \mathrm{m}(\mathrm{G})$, and $50 \mu \mathrm{m}(\mathrm{H})$.

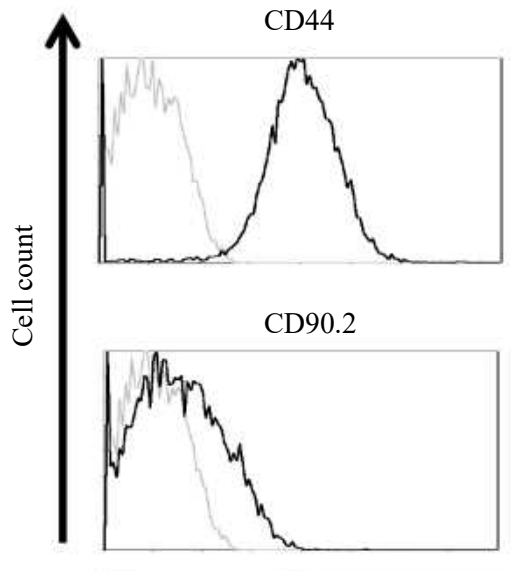

CD29

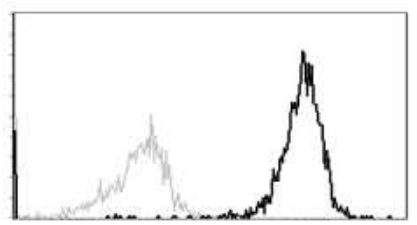

CD45

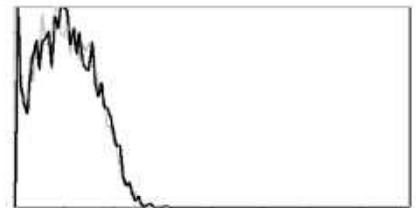

Intensity
Sca-1

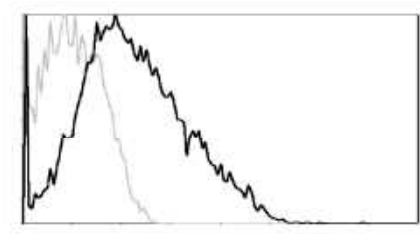

$\mathrm{CD} 11 \mathrm{~b}$

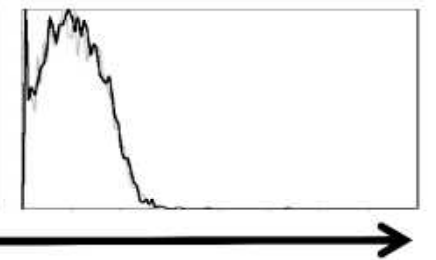

Figure 2. Representative flow cytometric analysis for indicated antibodies in iPS-NCLC-MSCs.

cells (Fig. 5A). Calcium deposition and OP expression were observed in iPS-NCLC-MSCs but not in cells in normal growth medium (Fig. 6A). After 2 weeks adipogenic induction, iPS-NCLC-MSCs showed increased expression of peroxisome proliferator-activated receptor gamma (PPAR $\gamma$ ) mRNA (Fig. 5B), as well as positive staining for oil red $\mathrm{O}$ and FABP (Fig. 6B). Chondrogenic differentiation was observed after culture as micromass pellets in medium containing chondrogenic supplements for 3 weeks. The pellets showed significantly upregulated expression of Col2 mRNA (Fig. 5C) and were alcian blue and Col2 positive (Fig. 6C), verifying a chondrogenic character. In addition, to determine the specificity of osteogenic induction of iPS-NCLC-MSCs, the mRNA expression of PPAR $\gamma$ and $\mathrm{Col} 2$ was evaluated in iPS-NCLCMSCs induced to osteogenesis. As expected, the expression of PPAR $\gamma$ and Col2 was not changed in osteogenic samples compared to iPSNCLC-MSCs in normal growth medium (data not shown).

\section{Osteogenic potential of iPS-MSC on hydroxyapatite}

We also tried to verify the capability of iPS-NCLC-MSCs to 
iPSs

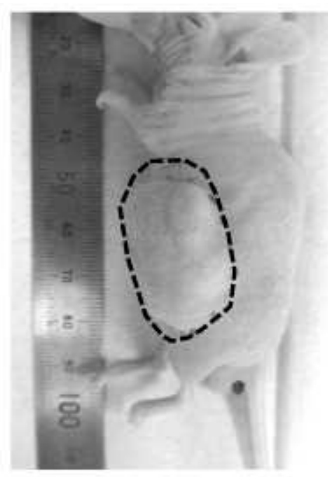

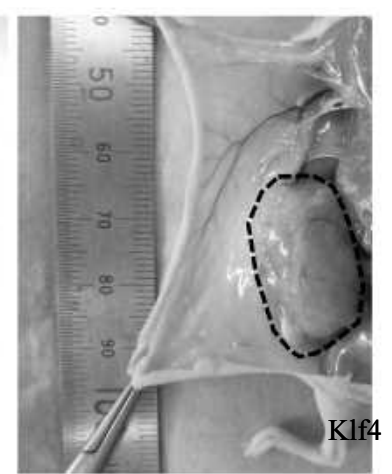

iPS-NCLC-MSCs

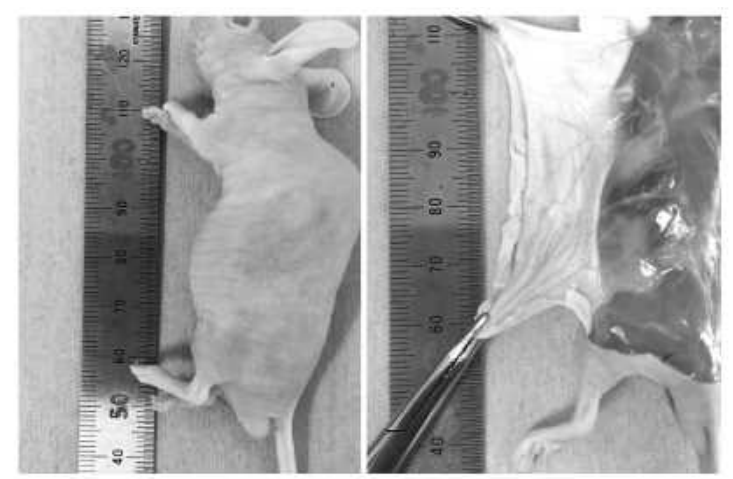

Figure 3. Suppression of tumorigenicity in iPS-NCLC-MSCs in vivo. Tumor monitoring 4 weeks after injection of undifferentiated iPS (iPS) (left) and iPS-NCLC-MSCs (right). The dotted circle indicates the teratoma.

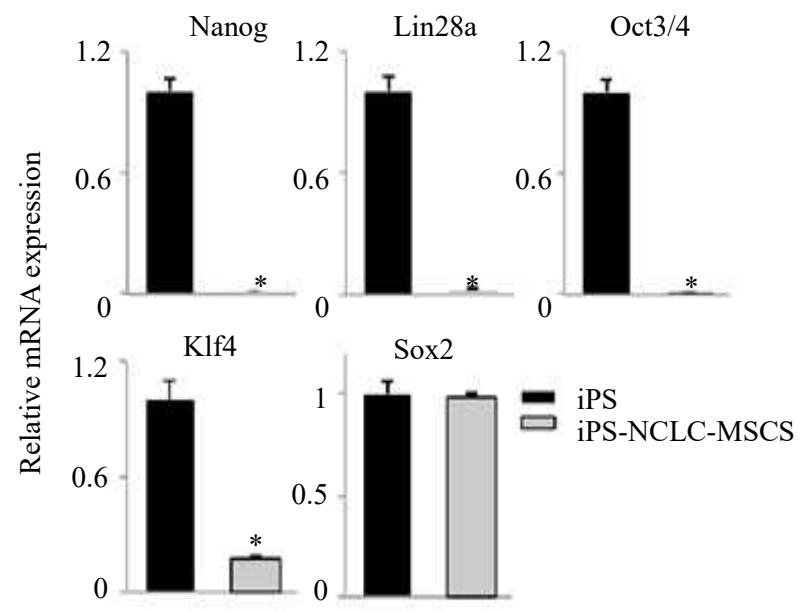

Figure 4. Expression of undifferentiated cell markers in undifferentiated iPS (iPS) and iPS-NCLC-MSCs determined by RT-qPCR. $\mathrm{n}=3$ per group, $* \mathrm{P}<$ 0.05 .

differentiate into osteoblasts on hydroxyapatite (HA), the major inorganic component of bone. GFP-expressing iPS-NCLC-MSCs were seeded in fibronectin pre-coated unidirectional porous HA scaffolds (UDPHAp) (Fig. 7A) and cultured for 2 days in normal growth media. Then the cells were cultured in osteoinduction media for 3 weeks. Immediately after seeding in the scaffold, the cells exhibited a round shape (Fig. 7Ba, arrows). After 2 days, the cells attached and grew on the HA and exhibited a spindle shape under fluorescence microscopy (Fig. 7Bb, arrows). After 3 weeks of osteoinduction, immunocytochemistry revealed that OSX was expressed in GFPexpressing iPS-NCLC-MSCs (Fig. 7C, arrows). OP was also distributed in the cells (Fig. 7D, arrows) and the edge of the scaffold (Fig. 7D, arrowheads), indicating that iPS-NCLC-MSCs could grow well and differentiate into osteoblasts on HA.

\section{Osteogenic potential of iPS-NCLC-MSCs in vivo}

To evaluate the osteogenic potential of iPS-NCLC-MSCs in vivo, GFP-expressing iPS-NCLC-MSCs were transplanted into criticalsize calvarial bone defects along with $\beta$-TCP and bone regeneration was assessed by histological observation. H\&E staining showed newly formed osteoid tissues around $\beta$-TCP in iPS-NCLC-MSCs-transplanted calvarial defects 8 weeks after transplantation (Fig. 8A, arrows) whereas this was rarely seen in controls (Fig. 8B). Immunohistochemistry
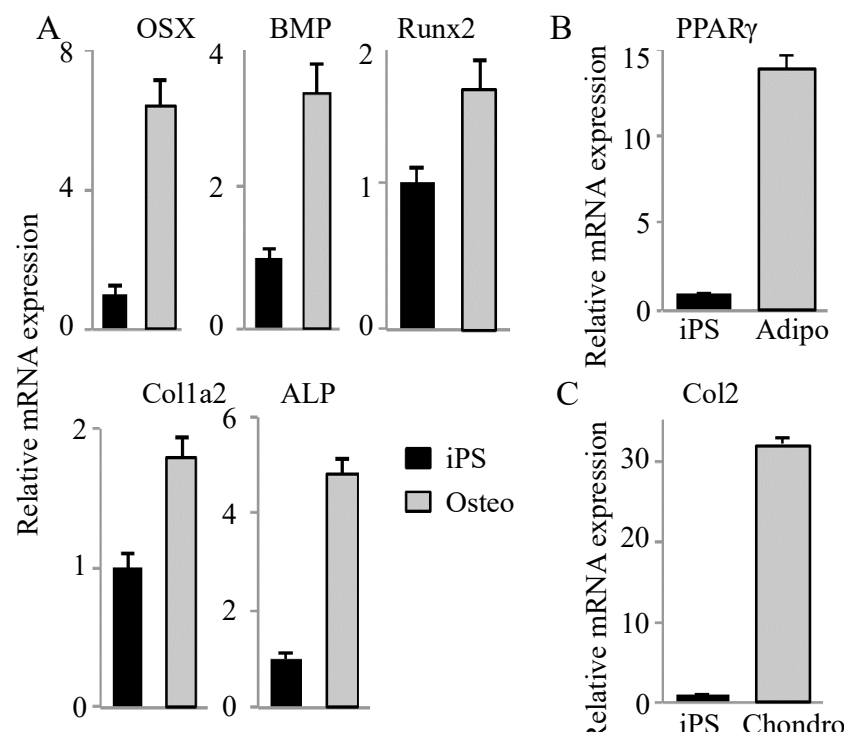

C

$\mathrm{Col} 2$

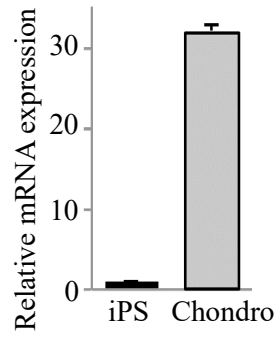

Figure 5. Expression of mesenchymal lineage-specific marker genes determined by RT-qPCR. Graphs show indicated mRNA expression in undifferentiated iPS cells and iPS-NCLC-MSCs after osteo- (A), adipo- (B), and chondro-induction $(\mathrm{C}) . \mathrm{n}=3$ per group, ${ }^{*} \mathrm{P}<0.05$.

demonstrated that the osteoid tissues were positive for OP, BSP, and ON (Fig. 8C). Further, the GFP-positive cells around the osteoid tissues expressed OSX, indicating that iPS-NCLC-MSCs differentiated into osteoblasts (Fig. 8D). Together, these results demonstrated that iPSNCLC-MSCs had the potential to contribute to bone regeneration in vivo.

\section{Discussion}

With increasing interest in stem cell therapy for diseases that cause bone loss, it is fundamental to obtain a readily accessible and safe stem cell source with an osteogenic ability. To date, many types of stem cells such as MSCs and ESCs have shown great advanced potential for bone regeneration ${ }^{24-26)}$. However, each cell has major drawbacks that are still unresolved and impeditive to clinical application. Consequently, development of a readily efficient method for deriving osteogenic cells from patients' iPS cells is necessary to overcome the limitation of such cells. In the present study, we demonstrated the craniofacial bone regeneration capacities of iPS-NCLC-MSCs from which a novel osteogenic cell source can be proposed for bone tissue engineering. 


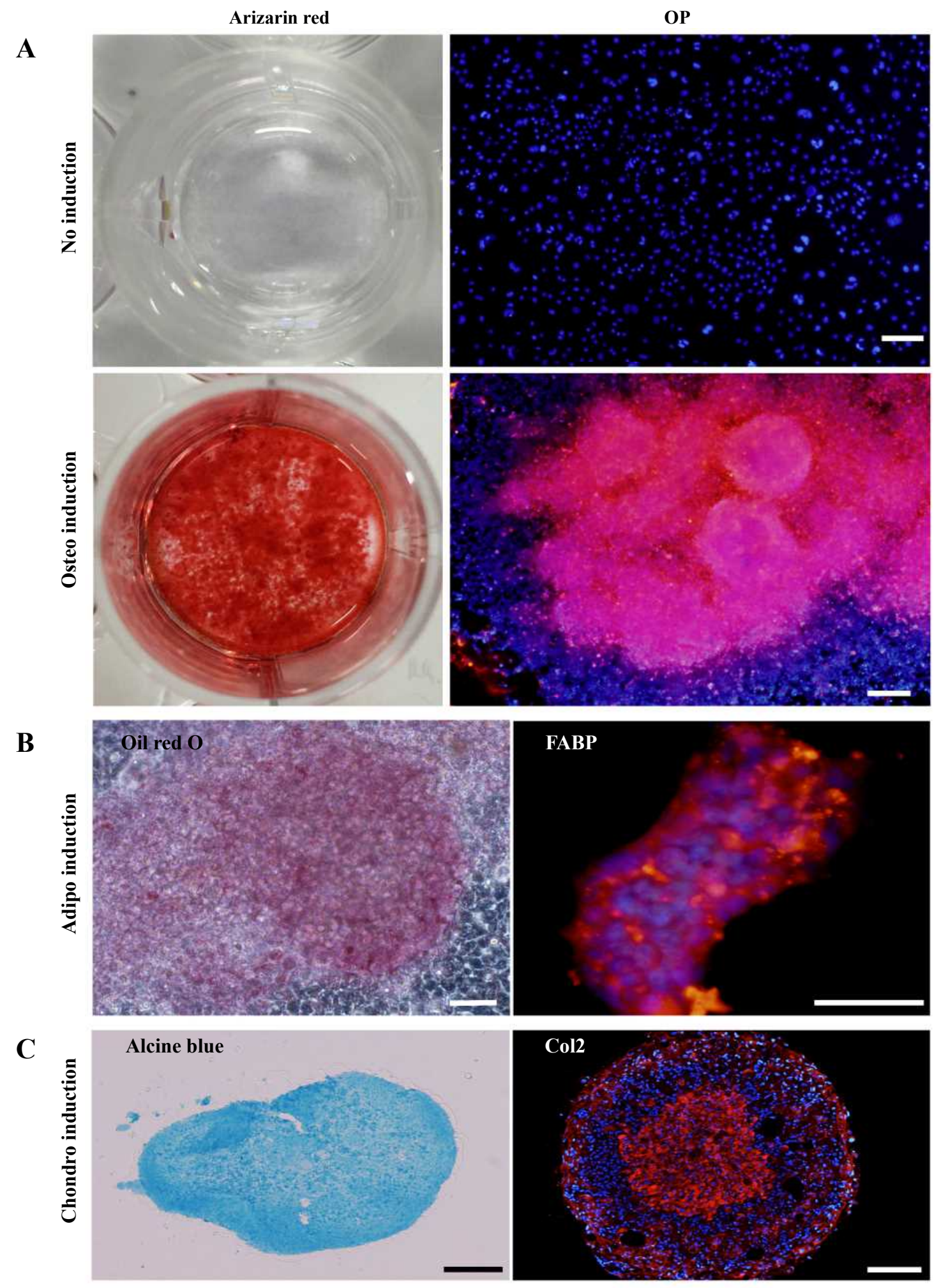

Figure 6. Mesenchymal lineage-specific staining of iPS-NCLC-MSCs after osteo-, adipo-, and chondro-induction. (A) iPS-NCLCMSCs were cultured with osteoinduction media. Osteogenic differentiation was detected by Alizarin red staining and osteopontin (OP) immunostaining. (B) Adipogenesis of iPS-NCLC-MSCs was detected by Oil red O staining and fatty acid-binding protein (FABP) immunostaining. (C) Differentiation into chondrocytes was detected by alcian blue staining and collagen type II (Col2) immunostaining. In immunofluorescence, nuclei were stained with DAPI (blue). Scale bar $=200 \mu \mathrm{m}(\mathrm{A}), 50 \mu \mathrm{m}(\mathrm{B})$, and $100 \mu \mathrm{m}(\mathrm{C})$. 
Kazuko Kikuchi et al.: Bone Regeneration by iPS-Derived Neural Crest Cells
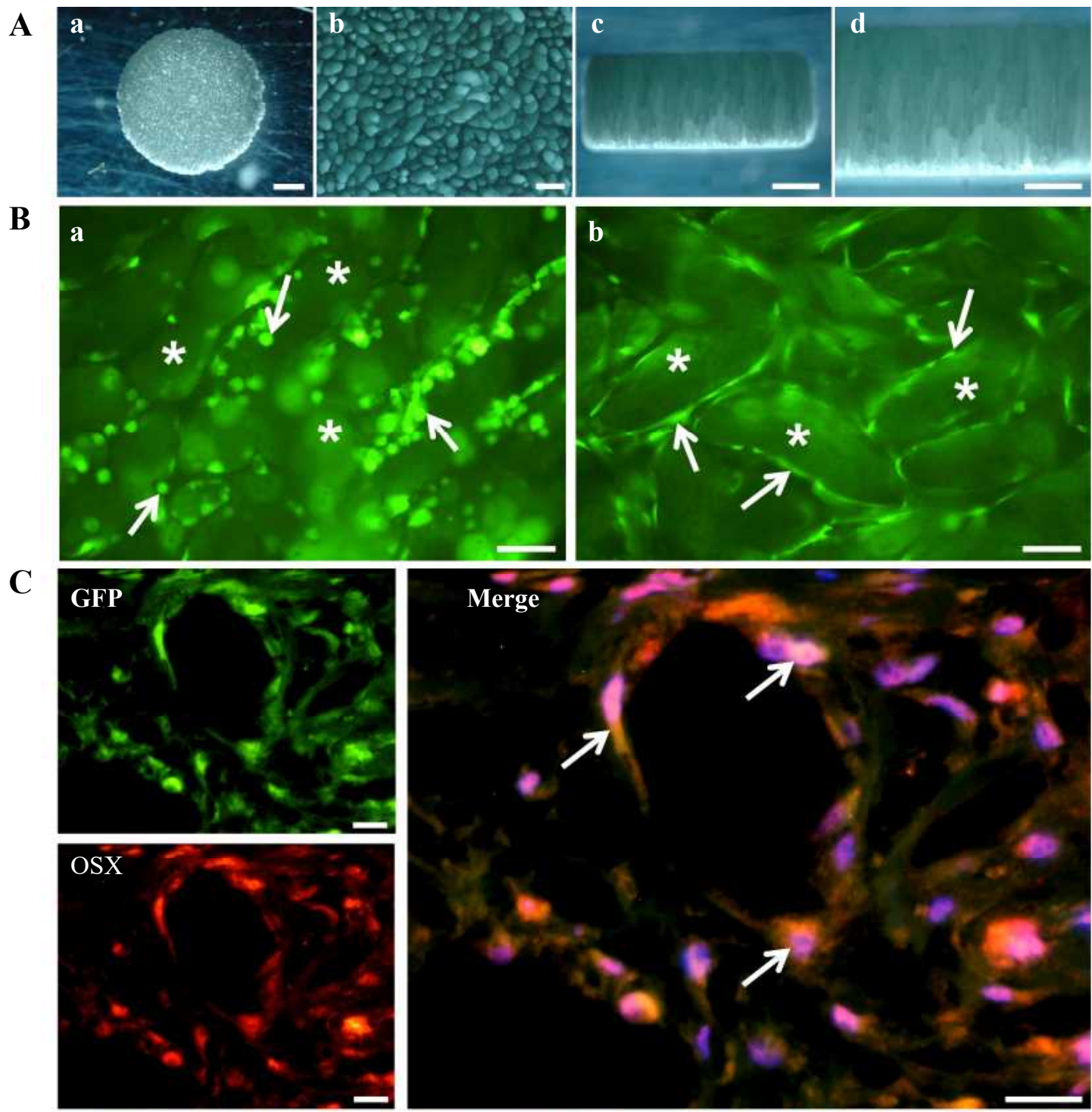

D
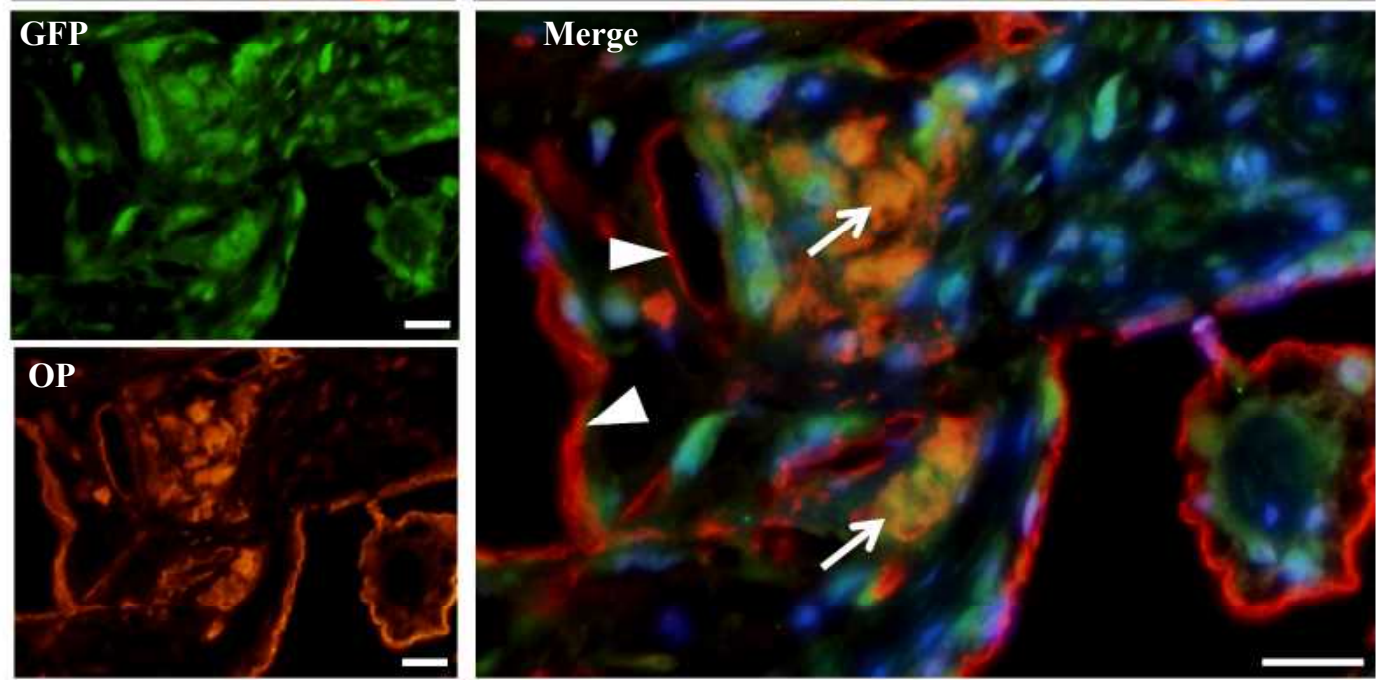

Figure 7. Osteogenic differentiation of iPS-NCLC-MSCs in UDPHA in vitro. (A) Representative digital microscopic photographs of UDPHA (a, b: horizontal dimension, c, d: vertical dimension). (B) GFP fluorescent images of iPS-NCLC-MSCs cultured in UDPHA $4 \mathrm{~h}$ after seeding (a) and 2 days of culture (b). Arrows indicate iPS-NCLC-MSCs; asterisks indicate porosity of UDPHA. Immunostaining for Osterix (OSX) (C) and osteopontin (OP) (D) of GFP-expressing iPS-NCLC-MSCs cultured in UDPHA with osteoinduction media for 3 weeks. Nuclei were stained with DAPI (blue). Scale bar $=1$ $\mathrm{mm}(\mathrm{Aa}, \mathrm{Ac}, \mathrm{Ad}), 200 \mu \mathrm{m}(\mathrm{Ab}), 100 \mu \mathrm{m}(\mathrm{B})$, and $20 \mu \mathrm{m}(\mathrm{C}, \mathrm{D})$. 
A

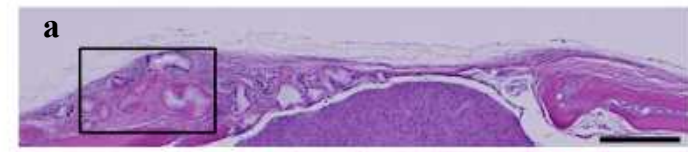

b

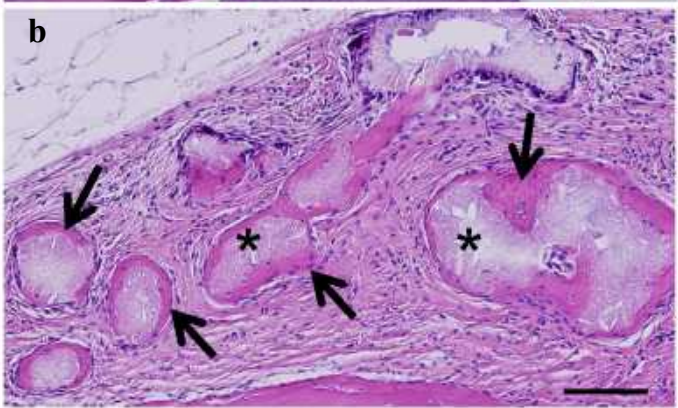

\section{B Control}
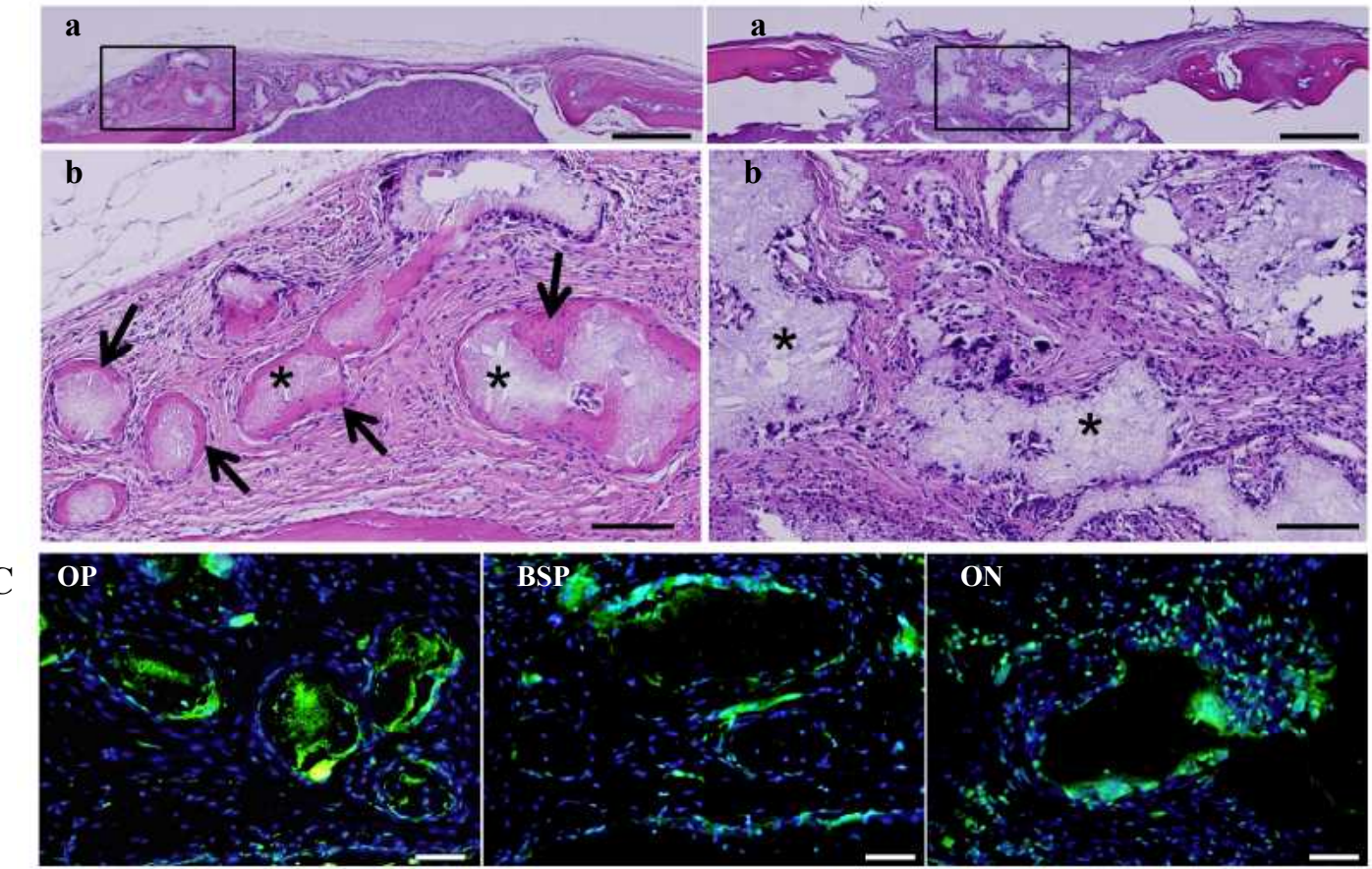

D
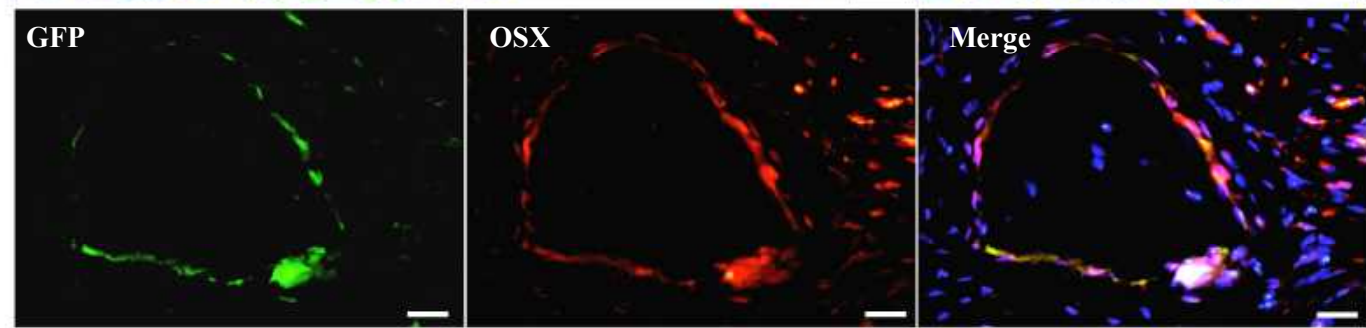

Figure 8 . Bone regeneration by iPS-NCLC-MSCs in critical-size calvarial defect in a mouse. Serial sections of the defect site were created at 8 weeks postoperatively. Represented slide of iPS-NCLC-MSCs (A) or no cell (B; control) transplanted calvarial defect was stained with hematoxylin and eosin. A-b and B-b are highly magnified images of the black squares in A-a and B-a, respectively. Newly formed osteoid tissues and transplanted $\beta$-TCP are denoted by arrows and asterisks, respectively. (C) Immunostaining for osteopontin (OP), bone sialoprotein (BSP), and osteonectin (ON) of iPS-NCLC-MSCs transplanted calvarial defect. (D) Newly formed osteoid tissues were double-stained with GFP and Osterix (OSX) antibodies. Nuclei are shown in blue. Scale bar $=500 \mu \mathrm{m}$ (A-a, B-a), $100 \mu \mathrm{m}$ (A-b, B-b), $50 \mu \mathrm{m}(\mathrm{C}), 20 \mu \mathrm{m}(\mathrm{D})$.

The most reasonable way to perform efficient organ/tissue regeneration is to follow the sequential developmental step in vivo. Thus, specific stem cells based on developmental research can help achieve successful tissue regeneration. In craniofacial development, the cranial neural crest $(\mathrm{NC})$ constitutes a major mesenchymal part of craniofacial tissues including bone ${ }^{27,28)}$. Indeed, a transgenic animal study using a lineage-tracing system experimentally showed that MSCs originated from NCs, and NC-derived cells resided in adult tissues with multipotent differentiation properties ${ }^{14-16,29,30)}$. Based on the developmental feature of the craniofacial bone, we explored the potential contribution of iPS-NCLCs ${ }^{12,13)}$ to craniofacial bone regeneration in this study. iPS-NCLCs cultured in serum-containing medium exhibited MSC phenotype expression of common MSC markers including Gli-1, craniofacial-specific MSC marker ${ }^{31)}$, and the multipotency to differentiate into 3 mesenchymal cell lineages. These findings were consistent with previous reports demonstrating that NCs derived from human iPS cells have a capacity to differentiate into $\mathrm{MSCs}^{32}$. iPS-NCLC-MSCs can also be cryopreserved and retain differentiation and proliferation capacity in long-term culture. These results indicate that iPS-NCLC-MSCs can overcome the problem of somatic tissue-derived MSCs. Notably, since our culture protocol to induce NCs from mouse iPS cells was modified originally from that of human ESCs, which is virtually equivalent to iPS cells ${ }^{33)}$, our protocol may be adaptable to human iPS cells. However, since detailed characteristics of derived iPS-NCLC-MSCs are still under investigation, further studies comparing the difference between iPS-NCLC-MSCs and somatic tissue-derived MSCs may provide more information to improve the efficiency of induction protocol.

For clinical application of pluripotent stem cells in tissue regeneration, tumorigenesis must be the most taken into account. In the present study, we proved that iPS-NCLC-MSCs had no tumorigenicity in vivo. The safety of iPS-NCLC-MSCs further confirmed the reduction of some pluripotency genes as shown in our previous study of iPS$\mathrm{NCLCs}^{12)}$. Consistent with our results, recent studies related to MSCs derived from iPS cells have demonstrated their safety for clinical application $^{32,34-36)}$, providing evidence to promote the use of iPS-NCLCMSCs for stem cell therapy. Interestingly, in iPS-NCLC-MSCs in the present study, Sox2 expression level was unchanged compared to that for undifferentiated iPS cells. Sox2 not only regulates the self-renewal of ESCs and plays an important role in the maintenance of adult 
stem cells, but also closely relates to many types of cancer in several tissues such as lung, esophagus, and breast ${ }^{37}$. Sox 2 also maintains the capability for expansion and differentiation of $\mathrm{MSCs}^{38)}$. However, the role of Sox 2 in the tumorigenesis of iPS cells is still unclear. In addition to Sox2, the relationship between other genes and tumorigenesis has been under debate. Zou et al. found that iPS-derived MSCs were positive for Nanog despite the exclusion of other pluripotent markers ${ }^{34}$. Lee et al. documented that polysialic acid-neural cell adhesion molecule (PSA-NCAM)-negative NC cells derived from human ESCs were tumorigenic ${ }^{39)}$. These findings suggest the importance and further necessity of basic and preclinical studies to evaluate the safety of the use of iPS cell-derived MSCs.

We further explored the possibility of clinical use of iPS-NCLCMSCs for craniofacial bone regeneration. First, we evaluated the engraftment and differentiation capacity of iPS-NCLC-MSCs on HA, the major mineral component of bone, and demonstrated that they exhibited the typical morphology of MSCs and could differentiate into osteoblasts by osteogenic induction. These results coincided with a previous study showing that MC3T3-E1 cells grew well on the UDPHAp surface ${ }^{20)}$ and suggested that iPS-NCLC-MSCs could maintain the differentiation capacity into osteoblasts on HA when transplanted. Second, we transplanted iPS-NCLC-MSCs with $\beta$-TCP in a calvarial defect and showed that the cells contributed to bone regeneration with no formation of tumor in vivo. In the implants, iPSNCLC-MSCs expressed osteoblast marker genes, indicating that the cells could differentiate into osteoblasts and directly contribute to bone regeneration in vivo. Recent reports have also shown that adult MSCs have the capacity to secrete factors such as soluble extracellular matrix glycoprotein, cytokines, and growth factors to modulate the microenvironment and recruit resident cells to repair the degenerated tissue $^{40)}$. Thus, transplanted iPS-NCLC-MSCs in this study may also play a role in activating endogenous osteogenic progenitor cells by secretion of such factors.

Here, we showed the efficient differentiation protocol from iPS cells into MSCs via NC induction, and that the iPS-NCLC-MSCs construct is promising to promote bone regeneration during calvarial bone repairs. Further study in other craniofacial implantation models such as periodontal and jawbone defects is required to validate its efficiency. Also, the functionality for vascularization and mineralization to form better bone as well as the safety issues of tumorigenicity and immune rejection should be carefully evaluated in the long term. In addition to a tissue regeneration standpoint, as bone formation by iPS-NCLS-MSCs in this study follows the developmental steps of craniofacial bone, this could be used to aid in the understanding of normal craniofacial bone development mechanisms and become a powerful tool for disease modeling and drug discovery.

\section{Acknowledgements}

This work was financially supported by JSPS KAKENHI Grant Numbers 24659819 and 21592500, JSPS Bilateral Joint Research Projects (2013-2015), Open Research Project Grant (2007-2011) from MEXT, and a Grant from Keiryokai Research Foundation No. 118.

\section{Conflict of Interest}

The authors have declared that no COI exists.

\section{References}

1. Damien CJ and Parsons JR. Bone graft and bone graft substitutes: A review of current technology and applications. J Appl Biomater 2: 187-208, 1991
2. Arrington ED, Smith WJ, Chambers HG, Bucknell AL and Davino NA. Complications of iliac crest bone graft harvesting. Clin Orthop Relat Res 329: 300-309, 1996

3. Joshi A and Kostakis G. An investigation of post-operative morbidity following iliac crest graft harvesting. Br Dent J 196: 167171, 2004

4. Kainer MA, Linden JV, Whaley DN, Holmes HT, Jarvis WR, Jernigan DB and Archibald LK. Clostridium infections associated with musculoskeletal-tissue allografts. N Eng J Med 350: 25642571, 2004

5. Parekkadan B and Milwid JM. Mesenchymal stem cells as therapeutics. Annu Rev Biomed Eng 12: 87-117, 2010

6. Kawano S, Otsu K, Kuruma A, Shoji S, Yanagida E, Muto Y, Yoshikawa F, Hirayama Y, Mikoshiba K and Furuichi T. ATP autocrine/paracrine signaling induces calcium oscillations and NFAT activation in human mesenchymal stem cells. Cell Calcium 39: 313-324, 2006

7. Ozawa K, Sato K, Oh I, Ozaki K, Uchibori R, Obara Y, Kikuchi Y, Ito T, Okada T and Urabe M. Cell and gene therapy using mesenchymal stem cells (MSCs). J Autoimmun 30: 121-127, 2008

8. Stenderup K, Justesen J, Clausen C and Kassem M. Aging is associated with decreased maximal life span and accelerated senescence of bone marrow stromal cells. Bone 33: 919-926, 2003

9. Kretlow JD, Jin Y-Q, Liu W, Zhang WJ, Hong T-H, Zhou G, Baggett LS, Mikos AG and Cao Y. Donor age and cell passage affects differentiation potential of murine bone marrow-derived stem cells. BMC Cell Biol 9: 60, 2008

10. Wagner W, Bork S, Horn P, Krunic D, Walenda T, Diehlmann A, Benes V, Blake J, Huber F-X, Eckstein V, Boukamp P and Ho AD. Aging and replicative senescence have related effects on human stem and progenitor Cells. PLoS One 4: e5846, 2009

11. Pittenger MF, Mackay AM, Beck SC, Jaiswal RK, Douglas R, Mosca JD, Moorman M A, Simonetti DW, Craig S and Marshak DR. Multilineage potential of adult human mesenchymal stem cells. Science 284: 143-147, 1999

12. Otsu K, Kishigami R, Oikawa-Sasaki A, Fukumoto S, Yamada A, Fujiwara N, Ishizeki K and Harada H. Differentiation of induced pluripotent stem cells into dental mesenchymal cells. Stem Cells Dev 21: 1156-1164, 2012

13. Otsu K, Kumakami-Sakano M, Fujiwara N, Kikuchi K, Keller L, Lesot $\mathrm{H}$ and Harada $\mathrm{H}$. Stem cell sources for tooth regeneration: current status and future prospects. Front Physiol 5: 36, 2014

14. Morikawa S, Mabuchi Y, Niibe K, Suzuki S, Nagoshi N, Sunabori T, Shimmura S, Nagai Y, Nakagawa T and Okano H. Development of mesenchymal stem cells partially originate from the neural crest. Biochem Biophys Res Commun 379: 1114-1119, 2009

15. Takashima Y, Era T, Nakao K, Kondo S, Kasuga M, Smith AG, and Nishikawa S-I. Neuroepithelial cells supply an initial transient wave of MSC differentiation. Cell 129: 1377-1388, 2007

16. Isern J, García-García A, Martín AM, Arranz L, Martín-Pérez D, Torroja C, Sánchez-Cabo F and Méndez-Ferrer S. The neural crest is a source of mesenchymal stem cells with specialized hematopoietic stem cell niche function. Elife 3: e03696, 2014

17. Okita K, Ichisaka T and Yamanaka S. Generation of germlinecompetent induced pluripotent stem cells. Nature 448: 313-317, 2007

18. Kashiwazaki H, Harada N, Akazawa T, Md Arafat K, Minamida $\mathrm{Y}$ and Murata $\mathrm{M}$. Bone augmentation using novel unidirectional porous hydroxyapatite with bone morphogenetic protein-2 on rat skull. J Hard Tissue Biol 22: 337-342, 2013 
19. Noguchi H, Watanabe A, Funayama T, Tsukanishi T, Wadano Y and Sakane M. A novel unidirectional porous hydroxyapatite cylinder implanted in the dorsal muscles of dogs promotes fibrous tissue vascularization and invasion. Key Eng Mater 529-530: 275-278, 2013

20. Tanaka M, Haniu H, Kamanaka T, Takizawa T, Sobajima A, Yoshida K, Aoki K, Okamoto M, Kato H and Saito N. Physicochemical, in vitro, and in vivo evaluation of a $3 \mathrm{D}$ unidirectional porous hydroxyapatite scaffold for bone regeneration. Materials 10: 33, 2017

21. Otsu K, Ida-Yonemochi H, Fujiwara N and Harada H. The semaphorin 4D-RhoA-Akt signal cascade regulates enamel matrix secretion in coordination with cell polarization during ameloblast differentiation. J Bone Miner Res 31: 1943-1954, 2016

22. Masuda T, Otsu K, Kumakami-Sakano M, Fujiwara N, Ema M, Hitomi J, Sugiyama Y and Harada H. Combined administration of BMP-2 and HGF facilitate bone regeneration through angiogenic mechanisms. J Hard Tissue Biol 24: 7-16, 2015

23. Cowan CM, Shi YY, Aalami OO, Chou YF, Mari C, Thomas R, Quarto N, Contag CH, Wu B and Longaker MT. Adipose-derived adult stromal cells heal critical-size mouse calvarial defects. Nat Biotechnol 22: 560-567, 2004

24. Bernardo ME, Pagliara D and Locatelli F. Mesenchymal stromal cell therapy: A revolution in Regenerative Medicine [quest]. Bone Marrow Transplant 47: 164-171, 2012

25. Bielby R C, Boccaccini A R, Polak J M and Buttery L D. In vitro differentiation and in vivo mineralization of osteogenic cells derived from human embryonic stem cells. Tissue Eng 10: 1518-1525, 2004

26. Buttery L, Bourne S, Xynos J, Wood H, Hughes F, Hughes S, Episkopou V and Polak J. Differentiation of osteoblasts and in vitro bone formation from murine embryonic stem cells. Tissue Eng 7: 89-99, 2001

27.Santagati F, Minoux M, Ren S-Y and Rijli F M. Temporal requirement of Hoxa2 in cranial neural crest skeletal morphogenesis. Development 132: 4927-4936, 2005

28. Chai Y, Jiang X, Ito Y, Bringas P, Han J, Rowitch D H, Soriano P, McMahon AP and Sucov HM. Fate of the mammalian cranial neural crest during tooth and mandibular morphogenesis. Development 127: 1671-1679, 2000

29. Neirinckx V, Coste C, Rogister B and Wislet-Gendebien S. Concise review: adult mesenchymal stem cells, adult neural crest stem cells, and therapy of neurological pathologies: a state of play. Stem Cells Transl Med 2: 284-296, 2013

30. Neirinckx V, Marquet A, Coste C, Rogister B and Wislet-Gendebien
$\mathrm{S}$. Adult bone marrow neural crest stem cells and mesenchymal stem cells are not able to replace lost neurons in acute MPTPlesioned mice. PLoS One 8: e64723, 2013

31. Zhao H, Feng J, Ho T-V, Grimes W, Urata M and Chai Y. The suture provides a niche or mesenchymal stem cells of craniofacial bones. Nat Cell Biol 17: 386-396, 2015

32. Fukuta M, Nakai Y, Kirino K, Nakagawa M, Sekiguchi K, Nagata S, Matsumoto Y, Yamamoto T, Umeda K, Heike T, Okumura N, Koizumi N, Sato T, Nakahata T, Saito M, Otsuka T, Kinoshita S, Ueno M, Ikeya M and Toguchida J. Derivation of mesenchymal stromal cells from pluripotent stem cells through a neural crest lineage using small molecule compounds with defined media. PLoS One 9: e112291, 2014

33. Bajpai R, Chen DA, Rada-Iglesias A, Zhang J, Xiong Y, Helms J, Chang C-P, Zhao Y, Swigut T and Wysocka J. CHD7 cooperates with PBAF to control multipotent neural crest formation. Nature 463: 958-962, 2010

34. Zou L, Luo Y, Chen M, Wang G, Ding M, Petersen CC, Kang R, Dagnaes-Hansen F, Zeng Y and Lv N. A simple method for deriving functional MSCs and applied for osteogenesis in 3D scaffolds. Sci Rep 3: 2243, 2013

35. Villa-Diaz LG, Brown SE, Liu Y, Ross A, Lahann J, Parent J M and Krebsbach PH. Derivation of functional mesenchymal stem cells from human induced pluripotent stem cells cultured on synthetic polymer substrates. Stem cells 30: 1174-1181, 2012

36. Xie J, Peng C, Zhao Q, Wang X, Yuan H, Yang L, Li K, Lou X and Zhang Y. Osteogenic differentiation and bone regeneration of iPSC-MSCs supported by a biomimetic nanofibrous scaffold. Acta Biomater 29: 365-379, 2016

37. Liu K-C, Lin B-S, Zhao M, Yang X-Y, Chen M, Gao A-d, Que J-W and Lan X-P. The multiple roles for Sox2 in stem cell maintenance and tumorigenesis. Cell Signal 25: 1264-1271, 2013

38. Go MJ, Takenaka C and Ohgushi H. Forced expression of Sox 2 or Nanog in human bone marrow derived mesenchymal stem cells maintains their expansion and differentiation capabilities. Exp Cell Res 314: 1147-1154, 2008

39. Lee Dongjin R, Yoo J-E, Lee Jae S, Park S, Lee J, Park C-Y, Ji E, Kim H-S, Hwang D-Y, Kim D-S and Kim D-W. PSA-NCAMnegative neural crest cells emerging during neural induction of pluripotent stem cells cause mesodermal tumors and unwanted grafts. Stem Cell Reports 4: 821-834, 2015

40. Spees J L, Lee R H and Gregory C A. Mechanisms of mesenchymal stem/stromal cell function. Stem Cell Res Ther 7: 125, 2016 